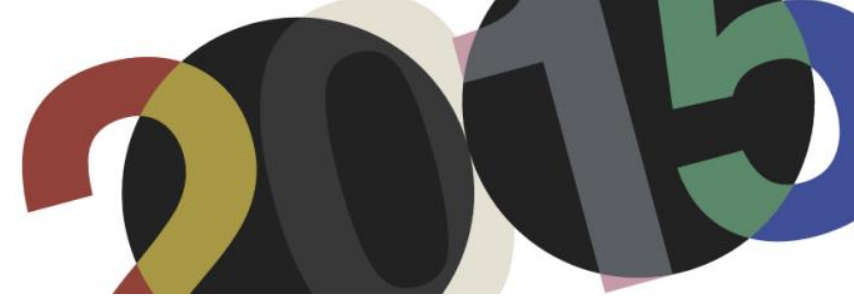

DOI: http://dx.doi.org/10.4995/LC2015.2015.880

\title{
Le Corbusier et le Brésil : une Synthèse des Arts Majeurs, et aussi des Arts Mineurs
}

\author{
C. Rodrigues dos Santos
}

Faculté d'Architecture et d'Urbanisme, Université Presbytérienne Mackenzie, SP, Brésil

Resumé: Ce travail examine les discours des architectes Lucio Costa et Le Corbusier sur la Synthèse des Arts, et les analyses faites à partir de l'application de ces idées dans les projets d'Architecture Moderne Brésilienne des années 1940, en particulier le projet du siège du Ministère de l'Éducation et de la Santé à Rio de Janeiro. L'objectif du présent travail est alors de révéler et de mettre l'accent sur la collaboration entre Le Corbusier et les architectes brésiliens ainsi que sur les Arts mineurs, représentés ici par l'art de l'azulejo, dans le processus de Synthèse des arts, comme supports et véhicules des expressions artistiques des Arts majeurs ou Beaux-arts.

Abstract: The objective of this work is, mainly through the speeches of Lucio Costa and of Le Corbusier on the "Synthèse des Arts" and the analysis of the application of these ideas to the works of the Modern Architecture in the years 1940 in Brazil, to reveal and to emphasize the important role of the Arts Mineurs and the Industrial Design in the process of the Synthesis of the Arts, as "vehicles" of the biggest expressions of the art intended to be integrated to the architecture, considering elements like ceramic tiles.

Mots-clés: Synthèse des Arts; Arts Majeurs et Arts Mineurs; Architecture Moderne Brésilienne. Keywords: Synthesis of the Arts; Major Arts and Mineurs Arts; Modern Brazilian Architecture. 
Le Corbusier entra en relation avec Lucio Costa et les architectes brésiliens lors de son premier voyage au Brésil, en 1929. Le pays vivait, à ce moment, un contexte de modernisation et de maturation de la discussion sur l'identité nationale ${ }^{1}$. Il s'agissait d'une relation complexe, qui se répercuta sur le travail du maître suisse et de ses disciples américains et fut bien plus profonde qu'une simple influence de Le Corbusier sur l'Architecture Moderne Brésilienne, comme l'a montré Carlos Eduardo Comas : "L'architecture de Le Corbusier ne se présente pas comme un système fermé, c'est comme un jeu de dominos. Ce que les Brésiliens Lucio Costa et Oscar Niemeyer font, c'est comprendre les règles de ce jeu, en capturer le mécanisme, la 'structure profonde' de l'auvre de Le Corbusier (...) ne signifie pas une soumission, mais une culture de l'identité (...). Les architectes brésiliens ont compris Le Corbusier suffisamment et ont été en mesure d'infléchir son travail, et l'inverse sera également vrai"'2; pour Lucio Costa, il s'agissait d'associer ses propres initiatives à celles de Le Corbusier, ayant en vue le renouveau de l'architecture brésilienne ${ }^{3}$. Le dialogue établi entre Costa et Le Corbusier sur les questions de la Synthèse des Arts, et la place que tous deux accordaient dans ce débat aux dits Arts Mineurs, n'est que l'un des chapitres de cette relation complexe et sophistiquée ${ }^{4}$.

Le déplacement de Le Corbusier de Paris à Rio de Janeiro, lors de son deuxième voyage au Brésil, en 1936 dure cinq jours. A bord du dirigeable Hindenburg, l'architecte écrit le texte "L'Architecture et les Arts Majeurs" "Les tendances de l'architecture rationaliste en rapport avec la collaboration de la peinture et de la sculpture. L'étude de la tendance qui règne, bien au contraire, dans l'architecture rationnelle, d'exclure, en tant que superflus d'après une logique rigoureuse, les concours des arts figuratifs" ${ }^{6}$. Ce texte ne sera publié, en portugais, qu'en 1984 par la Revista do Patrimônio Histórico e Artístico Nacional, avec préface de Lucio Costa, le gardien zélé du texte durant toutes ces années. L'article est traduit en portugais par Lucio Costa lui-même, qui l'intitule: "Arquitetura e as Belas-Artes"7 — "As tendências da arquitetura racionalista relativamente à colaboração da

\footnotetext{
${ }^{1}$ Sur les voyages de Le Corbusier au Brésil, voir : SANTOS, Cecilia R. dos ; PEREIRA, Margareth da S.; PEREIRA, Romão da S.; SILVA, Vasco C.. Le Corbusier e o Brasil. São Paulo, Projeto/Tessela, 1987.

2 BAPTISTA, Luis S.; VENTOSA, Margarida. "Prospectivas críticas - Herança de Le Corbusier", Architectura e Arte, $\mathrm{n}$. 50/60, julho/agosto 2008, Lisboa, Portugal.

${ }^{3}$ SANTOS, Cecilia. et alii. Op. cit., pp. 106-120.

${ }^{4}$ Lorsqu'on lui demanda pourquoi Le Corbusier avait reçu l'invitation pour aller au Brésil, en 1936, et pas un autre architecte en vue de cette époque, Walter Gropius par exemple, Lucio Costa expliqua: "Le Corbusier était le seul qui considérait le problème sous trois aspects : le sociologique (...), l'adaptation à la technologia nouvelle et l'approche plastique. C'est cela qui m'a frappé le plus, c'est là qu'il était différent de tous (...); l'approche de Le Corbusier séduisait beaucoup (...) il avait le don de la parole (...) et une foi énorme dans la rénovation, au bon sens du terme, une force qui contagiait les jeunes". Quand on lui demanda encore si Le Corbusier avait, d'une façon ou d'une autre, profité de son séjour au Brésil, Costa ajouta : "avec cette sensibilité impressionnante qu'il avait, il absorvait toujours quelque chose, dans n'importe quel pays. Sa richesse était là, exactement : il était sensible au régionalisme et était cosmopolita en même temps". In: "Presença de Le Corbusier" interview en 1987 à Jorge Czjkowsky, Maria Cristina Burlamaqui et Ronaldo Brito. In: COSTA, Lucio. Registro de uma vivência. São Paulo, Empresa das Artes, 1997, pp. 144-155.

${ }^{5}$ Le Corbusier arrive à Rio de Janeiro à l'aube du 13 juillet 1936, et reste au Brasil un peu plus d'un mois, jusqu'au 15 août. En 1929, il avait voyagé pour son compte, mais en 1936, "il répond objectivement, à une proposition de collaboration professionnelle avec des architectes locaux qui avaient déjà l'expérience d'un certain nombre de projets de caractère moderniste. Le Corbusier n'est donc pas venu au Brésil pour y fonder un nouveau mouvement architectonique, mais pour ratifier et d'une certaine façon accélérer le processus, déjà en cours, de rénovation de l'architecture brésilienne" (In: SANTOS, Cecilia et alii. Op. cit., p. 112). Le Corbusier fut invité par le Ministre de l'Éducation et de la Santé, Gustavo Capanema, à participer à un projet pour la Cité Universitaire de Rio de Janeiro et à donner une série de conférences à l'École des Beaux-arts; l'insistance de l'architecte lui-même lui permit de participer aussi, comme consultant, au projet du nouveau siège du Ministère de l'Éducation et de la Santé - MES.

${ }^{6}$ LE CORBUSIER. "A arquitetura e as Belas-Artes". Revista do Patrimônio Histórico e Artístico Nacional. Rio de Janeiro, IPHAN, n. 19 / 1984, pp. 53 a 69.

7 “L'Arts Majeurs" est traduit par Lucio Costa comme "Belas-Artes", "Beaux-Arts" en français.
} 
pintura e da escultura. $\mathrm{O}$ estudo da tendência que, ao contrário, impera na arquitetura racional de excluir como supérfluo, seguindo uma lógica rigorosa, o concurso das artes figurativas".

Les questions relatives à la Synthèse des Arts, discutées par Le Corbusier, sont tributaires des débats qui ont eu lieu en Angleterre au XIXe siècle, dans le cadre des Arts \& Crafts de W. Morris, ainsi que des propositions du premier Bauhaus et des discussions sur les métiers d'art ${ }^{8}$ et de la production industrielle en Allemagne, qu'il étudiera in situ pour faire publier, en 1912, le texte "Étude sur le mouvement d'art en Allemagne". En 1925, dans son livre Almanach d'Architecture Moderne, à propos du Pavillon de L’Esprit Nouveau, Le Corbusier écrit: "Nous ne sommes pas, à l' heure actuelle, partisans de la fresque, de la frise, de la métope. Il est mieux que l'ouvre peinte ou sculptée ne soit pas une 'commande' mais un produit direct de l'imagination. (...) Nous détachons du mur la sculpture et la peinture et les laissons seules, agir avec le radium qu'elles peuvent contenir"'. La même année, Le Corbusier publie le livre L'art décoratif d'aujourd'hui, sur les relations entre l'art (et l'architecture), le folklore et les arts décoratifs à "l'âge de la machine", esquissant les idées qui seront à l'origine du corpus de la Synthèse des Arts, mûries dans les œuvres ultérieures. En reprenant les registres de son voyage d’Orient en 1911, Le Corbusier définit des relations entre l'art décoratif, le décor, l'art figuratif, l'art industriel et le folklore (le folklore traditionnel et "notre folklore aujourd'hui", selon ses propres termes), pour indiquer quel décor serait compatible avec "les temps nouveaux", au détriment de l'art décoratif, de son déclin et de sa mort dont il se croit le héraut ${ }^{10}$.

Le décor ne devrait être envisagé, selon Le Corbusier, que s'il cherche son inspiration, sans imitations, dans les civilisations du passé légitimées par l'Histoire ainsi que dans la culture matérielle d'héritage européen représentée par des objets utilitaires d'origine archéologique (étrusques ou grecs, par exemple) ou d'origine régionale relatifs au folklore, aux arts populaires et à l'ethnographie (de la vallée du Danube ou de la Hongrie et de la Serbie, par exemple) $)^{11}$. Dans le dernier chapitre de L'Art décoratif d'Aujourd'hui, qu'il intitule "Confession", Le Corbusier publie une carte avec l'itinéraire de son voyage d'Orient, sous-titrée "Le voyage utile", où chaque destination porte les lettres $\boldsymbol{C}$ - Culture, ou $\boldsymbol{F}$ - Folklore ou $\boldsymbol{I}$ - Industrie; il explique : "L'architecture me fut révélée. L'architecture est le jeu magnifique des formes sous la lumière. (...) L'architecture n'a rien à voir avec le décor. L'architecture est dans les grandes œuvres, difficiles et pompeuses, léguées par le temps, mais elle est aussi dans la plus petite masure, dans un mur clôture, dans toute chose sublime ou modeste qui contient une géométrie suffisante pour qu'un rapport mathématique s'y installe. (...) Il n'y a pas de décor qui fasse jaillir l'émotion du voyageur : il y a l'architecture qui est forme pure, intégrale, - structure plastique -, et il y a les ouvres d'art : Phidias ou le pot du potier serbe des Balkans ${ }^{\prime \prime 2}$. Il appartient à l'architecture de créer l'atmosphère pour l'œuvre d'art, puisque son but est de "créer des rapports" ${ }^{13}$. Il ne s'agit pas encore d'une Synthèse, mais bien d'un rapport entre les arts. Et il ne s'agit pas d'un rapport entre les Arts Majeurs, puisque sont regroupées des œuvres qui vont des antiquités grecques aux céramiques serbes du XXe siècle. Lors de son passage en Hongrie, en 1911, Le

\footnotetext{
8 FERNANDES, Fernanda. "A síntese das Artes e a moderna arquitetura brasileira dos anos 1950". consulté: www.iar.unicamp.br/dap/vanguarda/.../fernanda_fernandes.pdf - ;voir aussi: "El concepto de Síntesis de las Artes" e "La irrupción de las sombras", consulté: www.tdx.cbuc.es/TESIS_UPC/...TDX...//04

${ }^{9}$ LE CORBUSIER, Almanach d'Architecture Moderne. Paris, G. Grès, 1925, pp.145-146.

${ }^{10}$ Voir aussi: TSIOMIS, Yannis. « Le Corbusier, L'Art décoratif d'aujourd'hui et 'La loi du ripolin' ».

${ }^{11} \mathrm{Au}$ sujet de la discussion sur l'ethnographie et le folklore et sur l'affirmation des identités nationales à cette époque en Europe et au Brésil, voir : SANTOS, Cecilia H G Rodrigues dos. Mapeando os lugares do esquecimento : ideias e práticas na origem da preservação do patrimônio no Brasil. Thèse de doctorat, Faculté d'Architecture et Urbanisme, Université de São Paulo, 2007.

${ }^{12}$ CORBUSIER. L'Art Décoratif d'Aujourd'hui. Paris, G. Grès, 1925, pp.210-217.

${ }^{13}$ Idem, p. 128.
} 
Corbusier annonçait déjà, dans une lettre aux amis de l'atelier d'art de La Chaux-de-Fonds, le devoir de fuir de "l'européanisation envahissante et sale" jusque dans les refuges tranquilles où survit la grande tradition populaire $^{14}$.

Le Corbusier s'intéresse au folklore qui met en valeur le travail anonyme, l'œuvre lointaine qui remonte à plusieurs siècles et se perfectionne sur l'échelle de l'émotion humaine pour devenir unanime et donc collective, "susceptible de l'universalité", pour, à son tour, "fournir sa sève" au travail individuel ${ }^{15}$. Le folklore, ainsi défini, apparaît comme "concis, bref, économique, intense, essentiel"l6, les mêmes attributs que Le Corbusier valorise dans le décor et dans l'architecture. L'objet contemporain moderne devrait être conçu selon un objectif et exécuté à la perfection en vue d'une utilisation certaine, pareil aux manufactures traditionnelles : "l'objet parfait est un organisme vivant, animé d'esprit de vérité". La critique de l'architecte se dirigée vers l'art décoratif, le "folklore usurpé par les paresseux et les stériles" pour être reproduit à grande échelle par la "machine à déformer", celle qui fabrique des objets qui semblent avoir été faits à la main, mais ne comptent plus ni avec "l inquiétude de l'inexplicable", caractéristique du folklore traditionnel, ni avec la "sérénité de la connaissance", attribut de l'art ${ }^{17}$. À Rio de Janeiro, en 1936, Le Corbusier note dans son carnet: "Il n'y a pas d'Art décoratif. Il y a l'art. L'art, c'est l'harmonie et non pas le décor. L'expression la plus désintéressée et la plus expressive du lyrisme se réalise dans les Arts Majeurs"18.

Selon la tradition des Beaux-Arts, seules l'architecture, la sculpture et la peinture méritent le titre d'Arts Majeurs, alors que les activités et les "choses" de la vie quotidienne, telles que les meubles, lampes, tissus, vaisselle, dont la confection est confiée à l'art des artisans ou des techniciens, rentrent dans la catégorie des Arts Mineurs. Pour Jenny Dreyfus ${ }^{19}$, la division et la hiérarchisation de l'art en Arts mécaniques ou industriels (concernant le travail manuel) et Arts libéraux (fruits de l'esprit et de l'imagination), vont provoquer la division, encore adoptée en France par l'Académie au moment où l'auteure écrit son livre (1946-1949), entre Arts Majeurs ou Beaux-Arts (y compris l'architecture -l'Art Majeur par excellence-, la sculpture et la peinture) et les Arts Mineurs ou arts appliqués ou arts rétrospectifs ou arts décoratifs: "Les Arts mineurs sont le résultat de produits normaux du travail humain, qui n'ont pas atteint la figure de proue des grands arts mais présentent cependant un aspect esthétique associé à un caractère d'utilité qui leur est essentiel"20. Aucune dépréciation des Arts Mineurs ne se justifierait, selon la muséologue, car ils renvoient à des artefacts qui eux aussi ont des qualités, telles que la solidité, la maîtrise de la forme et la perfection de la finition. Mais Jenny Dreyfus reconnaît que "l'art de la parure $^{\prime 21}$ peut être considéré comme inférieur dans la hiérarchie des Beaux-Arts, ainsi que les différents types d'objets considérés comme appartenant aux arts décoratifs, comme les meubles, l'argenterie, l'orfèvrerie, le

\footnotetext{
${ }^{14}$ LE CORBUSIER. Viagem do Oriente. São Paulo, Cosacnaify, 2007, pp. 17-28. Dans la présentation de l'édition de la Cosacnaify, en portugais, Margareth da Silva Pereira nous rappelle que ce livro, révisé par Le Corbusier l'année de sa mort, et dont la publication fut posthume, peut être considéré comme son premier et dernier livre, un texte qui nous fournit la clé pour comprendre l'œuvre de l'architecte.

${ }^{15}$ LE CORBUSIER. L'Art Décoratif d'Aujourd'hui. Paris, G. Grès, 1925, pp.33 et 211.

${ }^{16}$ Idem p. 32

${ }^{17}$ Ibidem, p. V et pp. 27-32.

${ }^{18}$ Notes sur feuilles séparées, numérotées de 1 à 6 , portant l'en-tête "Cabinet do Ministère de l'Éducation et de la Santé Publique”, Fondation Le Corbusier, A2-19. In: SANTOS, Cecilia et alii. Op. cit., p. 149

${ }^{19}$ DREYFUS, Jenny. Artes Menores. São Paulo, ANHAMBI, 1959. J. Dreyfus (1890-1977) a été conservatrice du Musée Historique National à Rio de Janeiro, et professeure de la matière "Arts mineurs" dans le Cours de Muséologie.

${ }^{20}$ Idem, p. 09

${ }^{21}$ Parure est le motif décoratif basé sur le dessin, qui sert à enjoliver ou orner des objets ou choses dans la structure desquels il soit intégré harmonieusement (In: DREYFUS. Op. cit., p. 15)
} 
textile, la tapisserie, la céramique, le verre, et encore les mosaïques, les carrelages, les vitraux - "tous les objets qui entourent l'homme et son foyer ${ }^{\prime 22}$.

Les arts appliqués à l'industrie ${ }^{23}$ - bronze, meubles, orfèvrerie, céramique, mosaïque, tapisserie, vitraux - étaient classés sans hésitation comme arts décoratifs ; cependant, lorsqu'il s'agissait de la peinture et de la sculpture, associées ou intégrées à l'architecture - sous forme de fresques, peintures murales, vitraux, ornementation architecturale, bas-reliefs, médaillons ou statues - l'encadrement comme art décoratif ou Art Mineur devenait plus difficile, ce que l'on découvre déjà dans les textes de Le Corbusier. Dans le chapitre "L'heure de l'Architecture", du livre L'Art décoratif d'Aujourd'hui, Le Corbusier fait l'histoire critique de la fin du XIXe siècle et, tout en y situant sa formation n'oublie pas d'en attribuer les crédits à Ruskin, Grasset, Owen Jones, William Morris et Walter Crane et de reconnaître dans leurs travaux "un superbe effort, un courage condidérable, une hardiesse très grande, une révolution véritable". Et il souligne encore «qu' "en 1900, l'incendie s'allume dans les esprits. On se met alors à parler d'Art décoratif. Et des escarmouches s'engagent : grand art, arts mineurs. Deux camps. $»^{24}$.

Dans l'article "La sainte alliance des Arts Majeurs ou le grand art en gésine", publié dans la revue La Bête Noire en juillet 1935, Le Corbusier annonce la possibilité d'une relation efficace entre les trois Arts Majeurs architecture, sculpture et peinture - et de ceux-ci avec les moyens d'expression ${ }^{25}$. En octobre 1936, peu après son retour du Brésil, il participe à Rome à la "Sixième Réunion VOLTA". Il y reprend le thème dans son texte intitulé "Les tendances de l'architecture rationaliste par rapport à la collaboration de la peinture et de la sculpture" ${ }^{26}$, où il met l'accent sur le parallélisme entre l'architecture et les deux autres Beaux-arts, et définit ce mouvement comme une "symphonie d'événements" et la "synthèse de la pensée" sur l'architecture ${ }^{27}$.

Le Corbusier ne reprendra ce débat que dix ans plus tard, après la fin de la guerre, dans le texte "L'Espace Indicible" publié dans la revue Architecture d'Aujourd'hui: "Ce texte doit être situé par le lecteur à sa juste place. L'an 1945 compte des millions de sinistrés sans abri, tendus désespérément vers l'espoir d'une transformation immédiate de leur situation. (...) Prendre possession de l'espace est le geste premier des vivants (...), manifestation fondamentale d'équilibre et de durée. (...) J'ignore le miracle de la foi, mais je vis souvent celui de l'espace indicible, couronnement de l'émotion plastique. (...) Architecture, sculpture, peinture, la marche du temps et des évènements les conduit indubitablement, maintenant, vers une synthèse" ${ }^{\prime 28}$. Deux ans plus tard, en 1948, il publie un autre article dans la même revue, intitulé "Unité" : "Le corps du domaine bâti est l'expression des trois arts majeurs solidaires". Et il ajoute, en expliquant que la Synthèse ne devrait pas être envisagée comme simple subordination de la peinture et de la sculpture à l'architecture, ni non plus comme subordination inverse : "Il s'agit davantage de trouver le moyen de parvenir à une unité sans compromettre l'autonomie de la construction, du tableau ou de la sculpture. Chacun des trois arts a sa propre fonction et

\footnotetext{
${ }^{22}$ DREYFUS, J.. Op. cit., ps. 09 e 10.

23 “Arts appliqués à l'industrie" est l'équivalent de "Industrial Design" en anglais.

${ }^{24}$ LE CORBUSIER. L'Art Décoratif d'Aujourd'hui. Paris, G. Grès, 1925, pp. 133-140.

25 Voir: "El concepto de Síntesis de las Artes" et "La irrupción de las sombras", consultés sur www.tdx.cbuc.es/TESIS_UPC/...TDX...//04 ; Voir aussi: Archives de la Fondation Le Corbusier, consulté sur : http://www.fondationlecorbusier.fr/ConfCONF1(2).htm

${ }^{26}$ MOOS, Stanislaus von. Le Corbusier - une synthèse. Marseille, Parenthèses, 2013, p. 317.

${ }^{27}$ LE CORBUSIER. «L'Espace Indicible ». Architecture d'Aujourd'hui, avril 1946. In : JENJER, Jean. Le Corbusier l'architecture pour émouvoir. Paris Gallimard, 1993, ps. 138-141.

${ }^{28}$ Idem
} 
poétique et aucun n'a besoin, pour se justifier, d'un accord symphonique dans l'œuvre d'art totale" 29 . Pendant l'automne 1949, par recommandation du CIAM VII (1947) au Congrès de Bridgewater en Angleterre, où l'on avait discuté de la Synthèse des arts, est fondée l'Association Synthèse des arts plastiques ${ }^{30}$. Cependant, en ce qui concerne le travail de l'architecte Le Corbusier, ces idées n'ont de répercussion que sur des fresques qui couvriront "les murs des maisons de certains de ses amis, profitant soit de l'admiration qu'ils lui vouent, soit de leur absence provisoire ${ }^{\prime 31}$. Comme, jusqu'en 1946, les grands projets de 1'architecte ne s'étaient pas concrétisés, il ne lui restait qu'à s'occuper de petits projets pour des maisons, des villas et pavillons, qui seront devenus les laboratoires de la Synthèse des Arts $^{32}$. En 1947, lorsque Le Corbusier entame la construction de l'Unité d'Habitation de Marseille, il s'interroge: "Où commence la sculpture? et la peinture? et l'architecture? ".

La réponse est donnée, cette même année, avec l'inauguration à Rio de Janeiro du siège du Ministère de l'Éducation et de la Santé - MES, une célébration compétente et précoce de la Synthèse des Arts, projet auquel Le Corbusier n'avait participé que comme consultant lors de son séjour au Brésil en $1936^{33}$. Parmi les nombreux principes corbuséens préconisés pour l'architecture moderne, le MES a inauguré la Synthèse des Arts en orchestrant le travail d'architectes (Oscar Niemeyer, Afonso Reidy, Jorge Moreira, Carlos Leão et Ernani Vasconcelos, sous la coordination de Lucio Costa), de sculpteurs (Jacques Lipchitz, Bruno Giorgi, Celso Menezes), du peintre Candido Portinari, de muralistes qui ont travaillé sur les panneaux de carrelage (Candido Portinari et Paulo Rossi Osir) et de l'architecte paysagiste Roberto Burle Marx ${ }^{34}$. Le ministre de l'Éducation et de la Santé, Gustavo Capanema, assume lui-même la défense de la Synthèse des Arts dans ce projet, dans une lettre qu'il adresse au président Getúlio Vargas, le 14 juin 1937. Le ministre y expose les raisons d'avoir fait appel à une collaboration entre sculpteurs et peintres pour la réalisation du projet pour le siège de son ministère ${ }^{35}$, ce qui, d'une certaine manière, rend "officielle" l'idée de la Synthèse des Arts au Brésil: "Les architectes, qui ont organisé le projet du palais pour le Ministère de l'Éducation et de la Santé, y ont mis de l'effort, du travail, du soin et du goût. Ils l'ont longuement fait et refait, afin de nous rendre finalement un travail sûr et beau (...) qui fonctionne de manière rationnelle (...), un modèle technique du bâtiment. Mais ils ont aussi voulu faire une ouvre d'art. Ce souci de faire de l'architecture, des ouvres d'art, n'a jamais cessé d'être un signe de la culture entre les nations. (...) Dès lors, on ne pouvait que les inviter à coopérer à la réalisation de l'ouvrage, de la sculpture et de la peinture. Ces deux arts sont complémentaires de l'architecture qui, à son tour, ne peut pas

\footnotetext{
${ }^{29}$ MOOS. S., op. cit., p. 319. Dans le même livre, en post-scriptum du chapitre 7 (pp. 372-375), Moos contextualise ce chapitre, "Éléments d'une synthèse", en expliquant que la première édition était sortie de presse quelques années à peine après la mort de Le Corbusier et que le sous-titre, "Éléments d'une synthèse" reflète encore la rhétorique optimiste du programme de l'architecte, en continuation directe de l'esprit des années cinquante. Les architectes modernes avaient dû relever alors un double défi : libérer l'architecture de son image techniciste et éviter les pièges des formules éculées de l'académicisme. La 'synthèse des arts' était à cet égard une alternative intéressante. Il s'agissait de retrouver une aura culturelle capable de replacer l'architecture sur le même plan que le monde de l'art et le mécénat de prestige.

${ }^{30}$ Henri Matisse était président de la section CIAM de Synthèse des Arts Plastiques, Le Corbusier était premier viceprésident et André Bloc deuxièmevice-président ; le siège social était celui de L'Architecture d'Aujourd'hui. In : RIKVIN, Arnoldo. "Un doble paradoxe". In : LUCAN, Jacques (org.). Le Corbusier - une encyclopédie. Paris, Centre Georges Pompidou, 1987, ps. 386-391.

${ }^{31}$ MOOS, S. Op. cit., p. 317.

${ }^{32}$ Idem, p. 319.

${ }^{33}$ À propos de la polémique suscitée par Le Corbusier au sujet du créateur du projet et des honoraires reçus, voir: SANTOS, Cecilia et alii. Op. cit., pp.106-217.

${ }^{34}$ Voir: SEGRE, Roberto. Ministério da Educação e Saúde - Ícone urbano da modernidade brasileira (1935-1945). São Paulo, Romano Guerra, 2013.

${ }^{35}$ On peut considérer comme certaine la participation de Lucio Costa à la rédaction de cette exposition des motifs signée par le ministre.
} 
exister sans eux pleinement. Les grandes époques de l'art montrent comment l'architecture, la sculpture et la peinture se sont réunies pour la composition d'une même ouvre. Ainsi ont été prévus par les architectes, lors du projet, quelques travaux qui sont strictement déterminés, de sculpture et de peinture. Ces œuvres n'ont pas été conçues de manière aléatoire, avec un souci d'ornement. Bien au contraire. Ils seront destinés à accorder à l'ensemble le signe de son destin, de sa finalité. (...) Ils ne seront donc non pas des artifices luxueux et inutiles, mais des parties complémentaires, conséquentes et nécessaires" ${ }^{36}$.

Si la Synthèse des Arts se concrétise pour la première fois au Brésil, dans le projet du siège du Ministère (19361945/1947), le débat à son sujet se définit et s'approfondit dans une étroite collaboration intellectuelle entre Lucio Costa et Le Corbusier. Les deux architectes, qui ont mûri ces questions dans leurs travaux et publications dès le milieu des années 1930, élargissent la discussion à la Conférence Internationale des Artistes, organisée par l'UNESCO à Venise, en 1952. Lucio Costa y présente "L'architecte et la société contemporaine"137 et donne comme référence le texte que Le Corbusier lui avait laissé au Brésil en 1936. Mais il cite bientôt son propre travail, "Raisons de la nouvelle architecture" ${ }^{138}$, qu'il date lui-même de 1933 à 1935: "l'œuvre d'art acquiert une voie précise et unanime : l'architecture, la sculpture et la peinture sont un ensemble cohérent, un organisme vivant dont la désagrégation est impossible". Le Corbusier, à son tour, présente à Venise une communication intitulée "Chantiers de synthèse des arts majeurs" ${ }^{139}$, qui se termine par la déclaration suivante: "Cette note n'a pas hésité à soutenir la communication de M. Lucio Costa, lue le 24 Septembre 1952 en session du Comité des Arts plastiques" ${ }^{40}$. À Venise, Lucio Costa prend son temps pour remettre en question "les agissements de certains peintres qui n'utiliseraient l'architecture que comme scénario de leurs œuvres, sans tenir compte d'une approche d'intégration" ${ }^{41}$, mais Le Corbusier préfère aborder la question en faisant appel à la reprise du contact de l'architecture avec les autres arts, à l'occasion d'une réunion qui ait lieu en faisant de l'associé une présence sur la réalité concrète du chantier. "Ouvrir des chantiers", répète-t-il avec insistance ${ }^{42}$.

Étant donné la participation de Lucio Costa au débat sur la Synthèse des Arts, et sa profonde connaissance de la langue française, nous pouvons dire que le choix du titre qu'il a donné au texte que lui avait confié Le Corbusier en 1936 n'a pas été accidentel ni occasionnel. Traduisant "Arts Majeurs" ${ }^{43}$ par «Belas Artes», "Beaux-Arts" en français, Lucio Costa semble d'une part chercher avant tout une association entre le grand débat qui se tient à l'époque en Europe et auquel Le Corbusier faisait référence dans son texte - "orner ou ne pas orner, décorer ou ne pas décorer, enrichir ou ne pas enrichir, ennoblir ou ne pas ennoblir, etc" - et d'un autre côté les tâches nécessaires pour intégrer les trois Beaux-Arts que Costa définira au long de ses réflexions: "l'intention plastique (...) est précisément ce qui distingue la construction simple de l'architecture (...), une fois établis les liens

\footnotetext{
${ }^{36}$ LISSOVSKY, Mauricio; SÁ, Paulo Sérgio Moraes de. Colunas da Educação - A construção do Ministério da Educação e Saúde. Rio de Janeiro, CPDOC/IPHAN, 1996, pp. 224-225.

${ }^{37}$ In: XAVIER, Alberto (org.). Lucio Costa: sobre arquitetura. Porto Alegre, Edit. UniRitter, 2007. D'après une note de l'organisateur, ce texte présente des extraits d'un autre texte de l'auteur "Considérations sur l'Art contemporain", daté de la même année.

${ }^{38}$ COSTA, Lucio. "Raisons de la nouvelles architecture". In: XAVIER, Alberto (org.). Lucio Costa: sobre arquitetura. Porto Alegre, Edit. UniRitter, 2007, p. 20.

${ }^{39}$ In: SANTOS, Cecilia; et alii. Op. cit., pp. 239 a 241.- Fundation Le Corbusier - U3.10.449/450.

${ }^{40}$ Idem

${ }^{41}$ FERNANDES, F., Op. cit

${ }^{42}$ SANTOS, Cecilia et alii. Op. cit., pp. 239 a 241.

${ }^{43}$ Majeur = plus grande; plus important; opposé a mineur. Dictionnaire de La Langue Française - Petit Robert.
} 
nécessaires d'intention en plastique avec les autres facteurs essentiels en cause (...), (elle) justifie sa classification traditionnelle dans la catégorie des beaux-arts" ${ }^{\prime 44}$.

Sans accepter la hiérarchie des arts dans sa traduction en portugais, et en refusant la classification des arts en majeurs et mineurs, Lucio Costa se permet d'introduire les questions "d'identité" et de "tradition" qui ne sont pas explicites mais à peine suggérées dans le discours écrit de Le Corbusier : "(...) Nous pouvons déjà voir des aspects "indigènes" de l'architecture moderne, d'apparence significativement différenciée quoiqu'obéissant aux mêmes principes de base et se servant de matériaux et de techniques communs. Non seulement parce que, sur les conseils de Le Corbusier lui-même, on peut voir la recherche délibérée de faire revivre, dûment intégrée à la nouvelle conception, l'expression de quelques souvenirs de parti général ou de détail de fond traditionnel toujours valables, et surtout parce que le caractère national lui-même s'exprime par la collaboration architecturale d'artistes authentiques, préservant ainsi ce qu'il y a d'impondérable, mais d'authentique et d'irréductible dans le caractère différent de chaque peuple ${ }^{\prime 45}$. Lucio Costa renforce donc l'appel insistant de Le Corbusier pour placer l'architecture "au-delà de l'utilité" ou l'importance de l'intention plastique en architecture, toujours rattachée à l'identité nationale. En même temps, Costa ouvre un espace qui permet de considérer quelle partie de la tâche de Synthèse entrerait dans les arts dits "artisanaux", "utilitaires" ou "mineurs", véhicules ou supports des expressions majeures qu'ils se proposent d'intégrer. Des éléments tels que azulejos ${ }^{46}$, mosaïques, fresques, vitraux, cobogós ${ }^{47}$, entre autres, qui sont des références dans les traditions coloniale et vernaculaire, fréquentent toujours davantage les travaux de l'architecture moderne brésilienne, ajoutant par ailleurs un sens de brésilienneté aux codes architectoniques de l'Architecture Moderne Internationale.

Parmi tous ces éléments, nous nous arrêterons ici à la fabrication traditionnelle de l'azulejo qui a été incorporé au projet du MES, par suggestion de Le Corbusier: "Ils ont entendu du professeur illustre les leçons qui tendent à mettre en valeur des matériaux locaux comme les granits cariocas à la place de marbres importés, plantes et arbres désuets comme le palmier royal (...), comme les azulejos qu'il avait appris à admirer lors de longues visites à l'église de la Glória do Outeiro" ${ }^{48}$. Dans son témoignage pour la revue Módulo, Lucio Costa confirme que la suggestion provenait de Le Corbusier, et qu'elle avait eu un accueil favorable de la part des Brésiliens: "Nous acceptons vos recommandations concernant la mise en place des azulejos dans les clôtures de rez-dechaussée et du gneiss dans les encadrements et les pignons". Costa expliquera plus tard qu'il avait envisagé d'utiliser dans le projet un genre de pierre de couleur paille-brûlée, le grès d'Ipanema, mais Le Corbusier avait insisté sur l'utilisation du gneiss - "ce granit est si beau, on dirait la peau d'un jaguar" - une pierre méprisée pendant la période coloniale au profit d'autres granits plus nobles, utilisée notamment pour paver certains

\footnotetext{
${ }^{44}$ COSTA, Lucio. "L’architecte et la société contemporaine" (en portugais). In: XAVIER, Alberto (org.). Lucio Costa: sur l'architecture. Porto Alegre, Editora UniRitter, 2007, p. 245.

${ }^{45}$ Idem, p. 243

${ }^{46}$ Le mot azulejo désigne en Espagne et au Portugal un carreau de faïence décorée, un carreau céramique. Simple matériau de couverture des surfaces murales, il est de plus investi d'ambitions artistiques.

${ }^{47}$ Le cobogó est un bloc décoratif ajouré, de béton ou de céramique, employé pour la construction de murs et de parois, qui filtrent le soleil et la luminosité ; développé dès le début du XXe siècle et largement utilisé dans l'architecture brésilienne moderne, il a comme références les muxarabis et les persiennes de l'architecturea traditionnelle.

${ }^{48}$ LEMOS, Carlos. "Azulejos décorés dans la modernité arquitectonique brésilienne". In: Revista do Patrimônio Histórico e Artístico Nacional. Rio de Janeiro, IPHAN, n. 20/1984, p. 171. Localisée au sommet d'une colline, l'église de Notre-Dame de la Glória do Outeiro fut terminée en 1739. Le projet est attribué au lieutenant-colonel José Cardoso Ramalho, et sa structure en pierre contraste avec le chaulage, avec les panneaux d'azulejos bleu-et-blanc à l'intérieur, attribués au maître en carreaux d'azulejo Valentim de Almeida, et avec la taille des retables. Consulté sur: http://outeirodagloria.org.br/igreja
} 
trottoirs de Rio de Janeiro et pour l'encadrement des portes des bâtiments du XIXe siècle ${ }^{49}$. Dix ans plus tôt, Le Corbusier avait encensé le folklore - "si intense le folklore, que tous, nous y sommes immédiatement sensibles (...), chose magnifique que le folklore (...), où le temps et la masse ont apporté la puretén ${ }^{\prime \prime 0}$ - attiré comme il l'était lors de ses voyages par "ces mélodies, ou alors ces vases, ces fétiches, ces maisons qui ne sont pas l'œuvre d'un certain Monsieur $X{ }^{51}$ et dont il étend les qualités au "folklore d'aujourd'hui", défini, comme le folklore traditionnel, comme "œuvre de perfection, digne et durable, conforme à nos besoins et répondant à notre pensér ${ }^{\prime \prime 52}$, et conforme aussi à l'économie, à la tradition et aux techniques de traitement des matériaux locaux. Pendant son séjour à Rio en 1936, Le Corbusier note dans un de ses carnets: "Au Brésil, recherche, étude et sauvegarde des folklores ${ }^{\prime \prime 53}$.

L'azulejo, une tradition dans les églises brésiliennes construites surtout dans le nord et le nord-est du Brésil au XVIIe et au XVIIIe siècles, ira également recouvrir au cours du XIXe siècle les façades des "sobrados", maisons urbaines à étages, surtout dans les villes du littoral. Plutôt que des motifs d'ordre esthétique, ce sont des raisons économiques et d'autres dues à la conservation et au confort thermique qui furent décisives dans ce choix, un fait qui, selon Costa, a particulièrement plu à Le Corbusier, qui observait attentivement les façades revêtues d'azulejos lorsqu'il se promenait à Rio de Janeiro. L'intérêt qu'il portait au granit et aux azulejos, encore fréquents dans l'architecture de Rio à cette époque, et la suggestion qu'il a faite que ces deux éléments figurent dans le projet du MES, sont enregistrés à trois reprises dans son carnet de voyage ${ }^{54}$. Paulo Osir, chargé de la fabrication de ces azulejos, confirme en avoir reçu une pièce, retirée de l'Eglise de la Glória ${ }^{55}$ - dont les panneaux magnifiques avaient particulièrement enchanté Le Corbusier - comme modèle du ton de bleu souhaité dans les panneaux conçus par Portinari. Et pour en finir avec les doutes concernant l'auteur de l'idée d'incorporer les références coloniales à l'architecture moderne du MES, par des matériaux et techniques des Arts Mineurs «d'appartenance nationale pure», Lucio Costa confirme : "Celui qui vient de l'étranger est toujours plus sensible et observe. Nous ne pensions pas à cela, ce n'était qu'un revêtement qui existait là. Et lui est arrivé avec la richesse d'une autre approche ${ }^{\prime 56}$.

D'origine arabe, l'azulejo est une plaque de format régulier, carrée ou rectangulaire, en argile cuite, dont une des surfaces est émaillée ou vitrifiée. À l'origine, la fabrication se développa notamment en Espagne, au Portugal, en Italie et plus tard aux Pays-Bas, grâce à l'amélioration des techniques traditionnelles de préparation et de la peinture, ainsi qu'à l'utilisation de la polychromie et des motifs figuratifs interdits chez les musulmans. Les premiers azulejos fabriqués au Portugal remontent au XVIe siècle et, dès le XVIIe, les pièces bleu-et-blanc commencèrent à prédominer en prenant la porcelaine chinoise comme modèle.

\footnotetext{
${ }^{49}$ In: Revue Módulo, n. 40, 1975, apud: SILVEIRA, Marcele Cristiane da. OL'azulejo dans la modernité architectonique (en portugais). Maîtrise, Faculté d'Architecture et Urbanisme - Université de São Paulo, 2008, p. 135.

${ }^{50}$ LE CORBUSIER. L'Art Décoratif d'Aujourd'hui. Paris, G. Grès, 1925, p.36.

${ }^{51}$ Idem, p. 33.

${ }^{52}$ Ibidem, p. 32 .

${ }^{53}$ Notes sur feuillets séparés, avec en-tête du "Cabinet do Ministère de l'Éducation et de la Santé Publique". In: SANTOS, Cecilia et alii. Op. cit., p. 150

${ }^{54}$ Sur les carnets de route, Rio de Janeiro : une page avec deux croquis pour l'édifice, et le commentaire : "Palais Ministère, granit, azulejos bleu-et-blanc", Carnet C12-736, In: SANTOS, Cecilia . Op. cit, p. 167; un croquis, vue générale avec l'église de la Glória, Carnet C12-754, In: SANTOS, Cecilia et alii. Op. cit. p. 116; un croquis de la latérale de l'église de la Glória avec indications sur le dessin pour du "gneiss rose" et "blanc", et le commentaire: "= force, + mathé, + diamant", Carnet C12-755, In: SANTOS, Cecilia et alii . Op. cit. p. 197.

${ }^{55}$ LISSOVSKY, M.; SÁ, P. S. M. Op. cit, , p. 272

${ }^{56}$ In: "Presença de Le Corbusier". Op. cit. ps. $144-155$
} 
Au Brésil, on trouve des azulejos surtout dans l'architecture coloniale de Bahia, Pernambouc, Maranhão et Rio de Janeiro ; pendant la période coloniale, la plupart des pièces venaient du Portugal. On les trouve avant tout dans les églises et les monastères, mais aussi dans des résidences, en panneaux de scènes religieuses ou profanes, ou comme "tapis". Au milieu du XIXe siècle, on commença à les appliquer comme revêtement des façades extérieures $^{57}$. Carlos Lemos et José Wasth Rodrigues s'accordent pour attribuer aux Brésiliens, et pas aux Portugais, l'idée de recouvrir d'azulejos les parois extérieures des maisons urbaines, en particulier celles situées à la côte, une tradition qui perd de sa force dès la moitié du XIXe siècle. Il s'agirait d'un choix motivé par les conditions climatiques, car l'azulejo est fait d'un matériau imperméable qui protège de l'humidité et diminue la température interne des immeubles ${ }^{58}$. Dans les premières décennies du XXe siècle, en réintégrant l'azulejo dans l'architecture, dans l'esprit des barres, des "tapis" et des médaillons d'architecture coloniale, et pas encore dans les façades revêtues, l'architecture néocoloniale fournit une demande nouvelle à une industrie de la céramique, jeune et sans l'expérience nécessaire pour faire face à un grand volume de combustion de pièces peintes à la main. Les panneaux d'azulejos de l'architecture néocoloniale privilégiaient des thèmes historiques et patriotiques, déjà marqués par un fort caractère "nativiste" 59 .

Suivant donc les indications de Le Corbusier, l'équipe d'architectes du bâtiment-siège du Ministère de l'Éducation et de la Santé décida d'inclure des parois d'azulejos sur certains murs non structuraux du rez-dechaussée. Ces panneaux seront le support de la nouvelle expression plastique de Candido Portinari, "les premiers azulejos qui marquent cette réviviscence d'un art presque abandonné ", selon les termes de Joaquim Cardozo ${ }^{60}$. Il existe sept panneaux d'azulejos dans le bâtiment du MES, "six sont dûs à Portinari, les deux principaux étant situés sur les façades du hall des fonctionnaires, l'un en face de la Rue Graça Aranha et l'autre sous les pilotis, face au hall principal. Le panneau de la cage de l'escalier secondaire fut conçu par Paolo Rossi, dont la firme Osirarte fabriqua tous les azulejos. (...) Portinari et Paulo Rossi choisirent des motifs marins comme thème des panneaux d'azulejos, peut-être parce que le bâtiment, à l'époque de sa construction, se situait en face de la Baie de Guanabara, car le remblai de Flamengo et les bâtiments frontaliers n'existaient pas encore" ${ }^{\prime 61}$.

Roberto Segre, qui a analysé la composition des panneaux, met en évidence les thèmes marins et les éléments unitaires, simples et répétitifs, poissons, étoiles de mer, sirènes, hippocampes, coquillages, escargots anthropomorphes, coraux ou crabes, ce qui permet à Portinari de créer sur les surfaces des panneaux de "maillages aux textures qui varient selon la prédominance d'une figure ou d'une autre, perceptibles comme formes sur un pan proche ou comme un tissu à distance" ${ }^{\prime \prime 2}$. Les azulejos créent un effet de dématérialisation des surfaces par le jeu de captation et réflexion de la lumière, qui accorde de la légèreté aux grande surfaces des murs et cloisons aveugles, pour recomposer plus tard, géométriquement, les mêmes surfaces à l'aide du traitement plastique, de la perspective et de l'application de la couleur. Grâce à l'azulejo, écrit G. C. Argan,

\footnotetext{
${ }^{57}$ RODRIGUES, José Wasth. Documentário Arquitetônico - Relativo à antiga construção civil no Brasil. São Paulo, Itatiaia - USP, 1979 , p. 224. Au cours de plusieurs années de voyages, d'études et de documentation de l'architecture coloniale au Brasil, José Wasth Rodrigues (1891-1957) réunit une collection de dessins d'édifices, de détails de constructions et d'éléments décoratifs, publiés dans cet ouvrage. Aux pages 224 à 227, il reproduit 23 modèles d'azulejos trouvés au Brésil, de diverses provenances.

${ }^{58}$ LEMOS, C., Op. cit., pp. 168 e 169.

${ }^{59}$ Voir: BREFE, Ana Claudia Fonseca. O Museu Paulista. São Paulo, Editora UNESP, 2003; KESSEL, Carlos. Arquitetura Neocolonial no Brasil. Rio de Janeiro, Jauá, 2008

${ }^{60}$ CARDOZO, Joaquim. Azulejos no Brasil, p. 106. Apud SEGRE, R. Op. cit., p. 431.

${ }^{61}$ RIBEIRO, Paulo Eduardo Vidal Leite. "Palácio Gustavo Capanema - Processo de restauração e revitalização" Consultado em :http://www.docomomo.org.br/seminario\%203\%20pdfs/subtema_B1F/Paulo_eduardo_ribeiro.pdf

${ }^{62}$ SEGRE, R. Op. cit., p. 414.
} 
"l'homme a restitué au ciel la lumière qui en provenait, mais remplie de sens humain". Il ajoute : "C'est un art des pauvres, la céramique : faite d'un peu de terre, d'un peu de couleur, et de feu. Mais ce n'est pas un art pauvre : plus simples sont sa matière et son artifice et plus somptueuse est sa vue. Mais c'est précisément parce que les matériaux sont pauvres que la splendeur de la couleur et de l'émail s'en trouve plus rehaussée. C'est presque une victoire de la richesse de la fantaisie sur la richesse matérielle" ${ }^{63}$.

Toutefois, comme le souligne Carlos Lemos, "en peu de temps (...), les raisons de Le Corbusier furent oubliées et de nombreux architectes modernistes, voire certains critiques, justifièrent rapidement l'utilisation d'azulejos décorés à peine comme éléments d'identification nationaliste, une question rattachée à l'identité culturelle" ${ }^{64}$. Associé à Oscar Niemeyer dans son bureau de São Paulo dans les années 1950, Lemos affirme que ce n'est pas cette idée qui aurait conduit l'architecte carioca, "conscient de ce qu'il faisait", à reprendre dans le complexe architectural de Pampulha ${ }^{65}$, à Minas Gerais, des panneaux aux azulejos bleu-et-blanc dessinés par Portinari. Lemos interrogea Niemeyer à ce sujet et celui-ci fit part de son désaccord avec ceux pour qui la présence de panneaux d'azulejos était presque devenue une "obligation moderniste", une "marque brésilienne indicative de notre architecture moderne, toujours répétée, associée à l'utilisation ici et là des treillis sur les vérandas et les balcons", traitée comme signe obligatoire de la modernité native. Niemeyer réaffirmera l'aspect rationnel et fonctionnel de l'utilisation d'azulejos sur les façades, comme un matériau qui résiste bien aux intempéries, sans mentionner des raisons pouvant suggérer des liens avec la tradition constructive brésilienne, ou comme signe obligatoire de la modernité native.

Pour la réalisation des grands panneaux d'azulejos du MES, Portinari va trouver Paulo Rossi Osir à São Paulo, et lui demande d'agrandir ses dessins sur des cartons et de les peindre sur les carreaux d'azulejos. Pour répondre à ce défi, l'artiste italo-brésilien crée, en 1940, l'Atelier d'Azulejos Osirarte, entreprise fondée pour fabriquer les pièces de revêtement de murs extérieurs du siège du Ministère ${ }^{66}$. En 1943, lors d'une exposition sur son travail, en fonction de cette première commande, Paulo Rossi explique: "Nous avons, bien sûr, une tradition d'azulejo, héritée des Portugais qui l'ont appliquée avec tant de soin à l'époque coloniale et qui s'est retransmise jusqu'à nos jours, au long de la période impériale et contemporaine (...). Mon ambition était, et est toujours, celle de suivre certaines lignes directrices, à la fois en ce qui concerne les questions techniques et les sujets, et de contribuer à créer un art décoratif à nous, indépendant, enraciné dans notre histoire, dans nos coutumes, bref, dans notre vie de tous les jours. Seul un ensemble d'artistes, dans une sorte de reconstitution de l'artisanat, $m$ 'a semblé en mesure d'accomplir ce projet" ${ }^{67}$.

Rossi décida d'utiliser la technique de peinture sur l'azulejo non émaillé pour la fabrication des 47.000 carreaux placés dans les panneaux du MES. Le dessin est d'abord exécuté sur la surface poreuse, qui absorbe l'encre très

\footnotetext{
${ }^{63}$ Lettre de G. C. Argan a Rafael Salinas Caiado. In: Revista Oceanos, n.36/37, octobre1998/mars1999, p. 235. Apud SILVEIRA, M. Op, cit., p. 125.

${ }^{64}$ LEMOS, C.. Op. cit., p. 171.

${ }^{65}$ L'ensemble architectonique de la Pampulha, à Belo Horizonte, Minas Gerais, fut un projet d'Oscar Niemeyer, à la suíte d'une commande du maire de la ville, Juscelino Kubitschek. Il fut construit entre 1942 et 1944. Il s'agit d'un ensemble de bâtiments, casino, église, salle de bal, club et hôtel entourant le lac artificiel de la Pampulha.

${ }^{66}$ Une série de commandes d'autres architectes et de particuliers, et la bonne acceptation des azulejos décoratifs créés par la firme Osirarte, prolongèrent l'existence de l'entreprise jusqu'à la fin des années 1950. Selon Paulo Almeida, “en un processus dialectique et entraînés par les propres courants rénovateurs, on cherchait un certain sens de modération et d'équilibre, en reprenant le fil des traditions légitimes, une fois rétablie la foi dans les connaissances techniques et dans le métier, comme éléments indispensables pour la réalisation d'œuvres d'art de longue vie" (ALMEIDA, Paulo Mendes de. De Anita au Musée. São Paulo, Perspectiva, 1976, p. 161).

${ }^{67}$ LISSOVSKY, M.; Sá, P. S. M. de. Op. cit., p. 272.
} 
rapidement, ce qui exige une grande exactitude du tracé. Les azulejos de 15 x $15 \mathrm{~cm}$ sont alors stockés puis envoyés à l'émaillage et la cuisson. De longues recherches et des essais effectués pendant trois ans dans le studio Osiarte témoignent de la mise en valeur du processus de fabrication et du métier de l'artiste : "Pendant quatre mois, j'ai mélangé des couleurs susceptibles de résister à des températures supérieures à 1.200 degrés, en essayant d'obtenir ce bleu intense particulier des azulejos portugais de l'église de la Glória. Mais je n'ai pas réussi. Mes recherches m'ont permis quand même de trouver un bleu intense, transparent et attrayant, qui a satisfait partiellement les exigences" ${ }^{68}$.

En 1953, un an après le Congrès de Venise, où se rendirent Le Corbusier et Lucio Costa, intéressés par le discours de la Synthèse des Arts, Max Bill, alors de passage à Rio de Janeiro, donna une interview au magazine Manchete, dans laquelle il critiqua l'architecture moderne brésilienne, en se référant aux panneaux d'azulejos du Ministère : "La beauté des plantes qui y sont présentes est plus que suffisante comme décoration. Les azulejos brisent l'harmonie de l'ensemble, ils sont inutiles et, de ce fait, ne devraient pas avoir été placés là. (...) Je suis contre la peinture murale dans l'architecture moderne. Le mural n'a eu sa raison d'être qu'à une époque où peu de gens savaient lire ; sa fonction a toujours été illustrative, c'est-à-dire de raconter par des images facilement reconnaissables ce que la plupart des gens ne pouvaient pas apprendre par la langue écrite (...), ce qui veut dire qu'il est inutile, et l'inutile est toujours antiarchitectural" ${ }^{\prime 9}$. Dans sa réponse au critique suisse, Lucio Costa se reporte tout d'abord à l'intégration des panneaux d'azulejos au projet du bâtiment, pour ensuite mettre en valeur cette solution, d'inspiration brésilienne, qui se sert de la tradition ancienne des panneaux d'azulejos comme support pour des œuvres d'art, enrobant les murs d'une œuvre de l'architecture moderne. (...). "Il trouve aussi inutiles et nuisibles les azulejos. Or, le revêtement d'azulejos au rez-de-chaussée et le sens fluide adopté dans la composition des grands panneaux ont la fonction très claire d'amortir la densité des murs afin de leur enlever toute impression de support puisque le bloc supérieur ne s'appuie pas sur eux, mais sur les colonnes. Puisque l'azulejo est un des éléments traditionnels de l'architecture portugaise, il était le nôtre, et il nous a paru opportun d'en renouveler l'application"70 .

La discussion tourne une fois de plus autour de la manufacture, de l'Art Mineur, critiqué ou prisé pour sa participation à l'Architecture Moderne Brésilienne, tantôt comme élément de la composition, ou comme technique de construction adaptée aux conditions tropicales, tantôt comme signe de brésilienneté ou comme référence à la tradition, ou encore por alimenter le travail des artisans. Pour Niemeyer, l'aspect d'imperméabilité et de confort thermique fournis par les revêtements d'azulejos s'avèrent prioritaires et sont les véritables signes de la modernité, qu'il faut transmettre ; et pour Lucio Costa, les panneaux d'azulejos du MES renforcent et surtout éclairent les caractéristiques structurelles et constructives du bâtiment. Les questions qui associent l'application des azulejos à celle des origines indigènes, à la récupération de l'architecture traditionnelle n'apparaissent qu'au deuxième plan, malgré tous les efforts demandés à l'Osiarte pour récupérer la technique de fabrication et l'intensité exacte du bleu d'origine. Peu à peu, va prévaloir le sens originel suggéré par Lucio Costa et par Le Corbusier, faire revivre, dûment intégrée à la nouvelle conception, l'expression de quelques souvenirs d'une partie générale ou d'un détail de fond traditionnel, toujours valable, expression propre du «caractère national ».

\footnotetext{
${ }^{68}$ Idem.

69 "Occasion perdue" (interview de Max Bill au magazine Manchete et réplique de Lucio Costa). In: XAVIER, Alberto (org.). Lucio Costa: sobre arquitetura. Porto Alegre, Editora UniRitter, 2007, ps. 252 a 259.

${ }^{70}$ Idem.
} 
Ainsi, en une véritable opération de Synthèse des Arts, des Arts Majeurs et ausi des Arts Mineurs, le bâtiment du MES inaugure cette importante série d'œuvres émaillées qui garnissent les murs extérieurs de ce monument, qui allait devenir l'un des principaux de l'Architecture moderne. Il s'agissait de mettre à jour l'emploi, la thématique, le dessin et les techniques de la fabrication traditionnelle des azulejos, un art mineur qui marque et distingue l'architecture portugaise et l'architecture brésilienne coloniale. Ensemble, architectes, artistes et aussi artisans, ont réalisé un grand projet de Synthèse des Arts, mais aussi d'affirmation de la modernité nationale. Si la voie de la Synthèse discutée par Le Corbusier et Lucio Costa conduisait bien à essayer de récupérer une source poétique commune pour les Arts Majeurs ${ }^{71}$, les raccourcis empruntés passeront loin des schémas beaux-arts, et récupéreront d'autres traditions comme références, toujours à la recherche d'une nouvelle unité pour les temps nouveaux. Concision, vérité, économie, intensité, essence, universalité figurent parmi les qualités identifiées par Le Corbusier et Lucio Costa dans les processus de création des expressions du folklore, du vernaculaire et des Arts Mineurs, autant de qualités qui finiront par atteindre les températures élevées de fusion nécessaires pour que la Synthèse des Arts soit accomplie, au-delà du discours. Dans un art dit "mineur", dont l'expression technique et esthétique est rattachée à la tradition, un rôle plus grand se révèle peut-être ici, même s’il n'est pas toujours reconnu et est souvent sous-évalué.

\section{Références Bibliographiques.}

ALMEIDA, Paulo Mendes de. De Anita ao Museu. São Paulo, Perspectiva, 1976.

BAPTISTA, Luis S.; VENTOSA, Margarida. "Prospectivas críticas - Herança de Le Corbusier”, Architectura e Arte, n. 50/60, julho/agosto 2008, Lisboa, Portugal.

BREFE, Ana Claudia Fonseca. O Museu Paulista. São Paulo, Editora UNESP, 2003.

CALATRAVA, Juan. «Un autre Le Corbusier : l’idée de la synthèse des arts majeurs ». La lettre du Collège de France, $\mathrm{n}^{\circ} 28,28$ avril 2010. Consultado em : http://lettre-cdf.revues.org/1049

COSTA, Lucio. "O arquiteto e a sociedade contemporânea". In: XAVIER, Alberto (org.). Lucio Costa: sobre arquitetura. Porto Alegre, Editora UniRitter, 2007.

COSTA, Lucio. "Razões da nova arquitetura". In: XAVIER, Alberto (org.). Lucio Costa: sobre arquitetura. Porto Alegre, Editora UniRitter, 2007.

DREYFUS, Jenny. Artes Menores. São Paulo, ANHAMBI, 1959.

"El concepto de Síntesis de las Artes", consultado em :www.tdx.cbuc.es/TESIS_UPC/...TDX...//04

FERNANDES, Fernanda. "A Síntese das Artes e a moderna arquitetura brasileira dos anos 1950". Consultado em: www.iar.unicamp.br/dap/vanguarda/.../fernanda_fernandes.pdf -

"La irrupción de las sombras", consultado em : www.tdx.cbuc.es/TESIS_UPC/.../TDX...//04

JENJER, Jean. Le Corbusier - l'architecture pour émouvoir. Paris, Gallimard, 1993.

JUNIOR, Juscelino H. C. Machado. A poética do vernáculo - Os paineis de Geraldo Queiroz no Triangulo Mineiro. Dissertação de mestrado, Instituto de Artes - Universidade Federal de Uberlandia, 2011.

KESSEL, Carlos. Arquitetura Neocolonial no Brasil. Rio de Janeiro, Editora Jauá, 2008.

LE CORBUSIER. "A arquitetura e as Belas-Artes". Revista do Patrimônio Histórico e Artístico Nacional. Rio de Janeiro, IPHAN, n. 19 / 1984.

\footnotetext{
${ }^{71}$ Voir aussi : CALATRAVA, Juan. "Un autre Le Corbusier : l'idée de la synthèse des arts majeurs". La lettre du Collège de France, $n^{\circ}$ 28, 28 avril 2010. Consulté sur : http://lettre-cdf.revues.org/1049.
} 
. A arte decorativa. São Paulo, Martins Fontes, 1996.

. «L'Espace Indicible », Architecture d'aujourd'hui, avril 1946. In : JENJER, Jean. Le Corbusier

- l'architecture pour émouvoir. Paris Gallimard, 1993, ps. 138-140.

A viagem do Oriente. São Paulo, Cosacnaify, 2007.

LEMOS, Carlos. "Azulejos decorados na modernidade arquitetônica brasileira". In: Revista do Patrimônio Histórico e Artístico Nacional. Rio de Janeiro, IPHAN, n. 20/1984.

LISSOVSKY, Mauricio; SÁ, Paulo Sergio Moraes de. Colunas da Educação - A construção do Ministério da Educação e Saúde (1935-1945). Rio de Janeiro, CPDOC/FGV-IPHAN, 1996.

MOOS, Stanislaus von. Le Corbusier - une synthèse. Marseille, Parenthèses, 2013.

"Oportunidade perdida" (entrevista de Max Bill à revista Manchete, e réplica de Lucio Costa). In: XAVIER, Alberto (org.). Lucio Costa: sobre arquitetura. Porto Alegre, Editora UniRitter, 2007.

“Presença de Le Corbusier" - entrevista de Lucio Costa a Jorge Czjkowsky, Maria Cristina Burlamaqui e Ronaldo Brito, 1987. In: COSTA, Lucio. Registro de uma vivência. São Paulo, Empresa das Artes, 1997, ps. 144 -155 .

RIBEIRO, Paulo Eduardo Vidal Leite. "Palácio Gustavo Capanema - Processo de restauração e revitalização" Consultado em:

http://www.docomomo.org.br/seminario\%203\%20pdfs/subtema_B1F/Paulo_eduardo_ribeiro.pdf

RIKVIN, Arnoldo. “Um doble paradoxe”. In : LUCAN, Jacques (org.). Le Corbusier - une encyclopédie. Paris, Centre Georges Pompidou, 1987.

RODRIGUES, José Wasth. Documentário Arquitetônico - Relativo à antiga construção civil no Brasil .São Paulo, Itatiaia - USP, 1979.

SANTOS, Cecília Rodrigues dos. "A Arquitetura e as Artes Menores". Atas/Proceedings VIII SEMINÁRIO DOCOMOMO BRASIL - Cidade Moderna e Contemporânea: Síntese e Paradoxo das Artes. Rio de Janeiro, 2009.

Mapeando os lugares do esquecimento: ideias e práticas na origem da preservação do patrimônio no Brasil. Tese doutoramento, Faculdade de Arquitetura e Urbanismo Universidade de São Paulo, 2007.

SANTOS, Cecilia Rodrigues dos; PEREIRA, Margareth da Silva; PEREIRA, Romão da Silva; SILVA, Vasco Caldeira da. Le Corbusier e o Brasil. São Paulo, Projeto/Tessela, 1987.

SILVEIRA, Marcele Cristiane da. O azulejo na modernidade arquitetônica. Dissertação de mestrado, Faculdade de Arquitetura e Urbanismo - Universidade de São Paulo, 2008.

TSIOMIS, Yannis. « Le Corbusier, L'Art décoratif d'aujourd'hui et 'la loi du ripolin' ». Consultado em maio de 2015, em : http://books.openedition.org/pupo/2422.

XAVIER, Alberto (org.). Lucio Costa: sobre arquitetura. Porto Alegre, Editora UniRitter, 2007.

http://www.fondationlecorbusier.fr/ConfCONF1(2).htm

http://www.itaucultural.org.br/aplicExternas/enciclopedia_IC/index.cfm?fuseaction=termos_texto\&cd_verbete= 334 